\title{
Effects of erythropoietin preconditioning on rat cerebral ischemia-reperfusion injury and the GLT-1/GLAST pathway
}

\author{
DAIHUA YU ${ }^{1 *}$, YUANHUA FAN $^{2 *}, \mathrm{XUDE} \mathrm{SUN}^{1}, \mathrm{LINONG} \mathrm{YAO}^{1}$ and WEI CHAI ${ }^{1}$ \\ ${ }^{1}$ Department of Anesthesiology, Tangdu Hospital of the Fourth Military Medical University, Xi'an, Shanxi 710000; \\ ${ }^{2}$ Intensive Care Unit, Ganzhou People Hospital, Nanchang University, Ganzhou, Jiangxi 325000, P.R. China
}

Received August 16, 2014; Accepted May 12, 2015

DOI: $10.3892 /$ etm.2015.2919

\begin{abstract}
The aim of the present study was to investigate whether erythropoietin (EPO) preconditioning affects the expression of glutamate transporter 1 (GLT-1) and glutamate aspartate transporter (GLAST) and protects against rat cerebral ischemia-reperfusion injury. A total of 140 Sprague Dawley rats were randomly assigned to one of the following four groups: Sham, EPO-sham, middle cerebral artery occlusion (MCAO) and EPO-MCAO. Neurological function scores were obtained 24, 36 and $72 \mathrm{~h}$ after reperfusion. Seventy-two hours after the induction of cerebral ischemia-reperfusion, the number of apoptotic neural cells and the cerebral infarct volume of each group were measured. The mRNA levels of GLT-1 and GLAST were determined using reverse transcription-quantitative polymerase chain reaction (RT-qPCR) analysis, while the GLT-1 and GLAST protein levels were assessed using western blotting. The cerebral infarct volume was significantly increased in the MCAO group compared with that in the sham group $(\mathrm{P}<0.01)$; however, the infarct volume of the EPO-MCAO group was significantly lower than that of the MCAO group $(\mathrm{P}<0.01)$. In addition, the number of apoptotic cells found in the MCAO group was higher than that in the sham group $(\mathrm{P}<0.01)$, but the number of apoptotic cells in the EPO-MCAO group was significantly lower than that in the MCAO group $(\mathrm{P}<0.01)$. The GLT-1 and GLAST mRNA
\end{abstract}

Correspondence to: Dr Daihua Yu, Department of Anesthesiology, Tangdu Hospital of the Fourth Military Medical University, 569 Xinsi Road, Xi'an, Shanxi 710000, P.R. China

E-mail: yudaihua@hotmail.com

Abbreviations: EPO, erythropoietin; GLT-1, glutamate transporter 1; GLAST, glutamate aspartate transporter; MCAO, middle cerebral artery occlusion; CCA, right common carotid artery; ECA, right external carotid artery; ICA, internal carotid artery; PPA, pterygopalatine artery; IZ, infarct zone

*Contributed equally

Key words: erythropoietin, cerebral ischemia-reperfusion, glutamate transporter-1, glutamate aspartate transporter and protein levels were significantly decreased $72 \mathrm{~h}$ after the cerebral ischemia-reperfusion $(\mathrm{P}<0.01)$ compared with those in the sham group, whereas the same levels were increased significantly in the EPO-MCAO group relative to those in the MCAO group $(\mathrm{P}<0.01)$. In conclusion, EPO preconditioning protected against cerebral ischemia-reperfusion injury and upregulated the GLT-1 and GLAST expression.

\section{Introduction}

Cerebral ischemia-reperfusion injury often occurs following the restoration of blood flow in cerebral stroke patients, and causes neurological deficits $(1,2)$. Despite the fact that great progress has been made over the years in studies of cerebral ischemia-reperfusion injury, numerous unsolved clinical problems remain. It is, therefore, of great importance to explore novel drugs that could contribute to the prevention and/or treatment of this condition.

Cerebral ischemia-reperfusion injury is closely associated with increases in the extracellular glutamate concentration, glial cell swelling and neuronal necrosis (3-5). Glutamate transporter 1 (GLT-1) and glutamate aspartate transporter (GLAST) are cation-dependent glutamate transporters, which not only transfer glutamate into glial cells, but also critically maintain appropriate glutamate gradients across intra- and extracellular environments. Ischemia- and hypoxia-induced astrocyte swelling may therefore be associated with GLT-1 and GLAST dysfunction, occurring due to the loss of glutamate balance between the inside and outside of the cell (6-8). Erythropoietin (EPO) reduces the extracellular glutamate concentration, which is increased by ischemia and hypoxia, and the glutamate-induced neural cell death (9). Furthermore, EPO has been shown to protect against cerebral ischemia-reperfusion injury in both experimental and clinical research $(10,11)$; however, little is known about the involvement of GLT-1 and GLAST in the protective effect of EPO against cerebral ischemia-reperfusion injury.

We hypothesized that EPO would upregulate the GLT-1 and GLAST expression to promote the transport of glutamate into astrocytes, thereby reducing the extracellular glutamate concentration and excitatory glutamate neurotoxicity induced by cerebral ischemia-reperfusion injury. The aim of the present study was to explore the protective effect of EPO against rat 
cerebral ischemia-reperfusion injury and its effect on the GLT-1 and GLAST expression.

\section{Materials and methods}

Animals. This study was approved by the Ethics Review Board of Tangdu Hospital (Xi'an, China; no. 2011036). A total of 140 male Sprague Dawley rats with an average body weight of 320-350 g were included in the present study and were randomly and evenly allocated into the following four groups: Sham (control group; neither occlusion nor reperfusion was performed), EPO-sham [neither occlusion nor reperfusion was performed but the rats received an intravenous injection of EPO (Sigma-Aldrich, St. Louis, MO, USA) at a dosage of $5,000 \mathrm{U} / \mathrm{kg}$ body weight], middle cerebral artery occlusion (MCAO; blood perfusion was restored $2 \mathrm{~h}$ after the MCAO) and EPO-MCAO (the rats received an intravenous injection of EPO 15 min prior to the MCAO at a dosage of $5,000 \mathrm{U} / \mathrm{kg}$ body weight and blood perfusion was restored $2 \mathrm{~h}$ later).

MCAO model. Rats were anesthetized by an intraperitoneal injection of $10 \%$ chloral hydrate $(350 \mathrm{mg} / \mathrm{kg}$ body weight) and fixed on the operating table in a supine position. A 2-cm incision was made in the middle of the neck and the right common carotid artery (CCA) was isolated so that the proximal portion could be ligated. The right external carotid artery (ECA) and internal carotid artery (ICA) were then isolated and the pterygopalatine artery (PPA) was isolated along the ICA without ligation. The ECA was ligated near the branching point of the CCA. The distal part of the CCA was nipped with a bulldog clamp. A loosely knotted silk thread was placed at the distal part of the CCA and a tiny incision was made at the bottom of the thread where a plug thread was inserted. The silk thread was then tightened to prevent the plug thread from slipping out and causing subsequent hemorrhage. The bulldog clamp was loosened and the plug was threaded into the calvarium, along the CCA and through the ICA. When a drag force was encountered, the plug thread was slightly retracted. The insertion depth of the plug thread was 17.5-40.5 mm away from the branching point so that it was located at the start of the MCA and blocked blood flow. The incision was then sewn up. Animals that woke up with the following four signs were used for further study: i) Adduction of the right forelimb with flexion when the tail was lifted up; ii) ipsilateral Horner's sign; iii) crawling to the right in circles and iv) falling down to the right upon standing. Animals in the control group underwent isolations of the CCA, ICA and PPA without ligation. Filament lamps were used to keep the animals at a normal body temperature.

Assessment of functional neurological deficit. Functional neurological deficit scores were obtained from animals 24 , 36 and $72 \mathrm{~h}$ after reperfusion (12) based on the following scoring system: Score 0, no functional neurological defect; score 1, failure to fully extend the right forepaw; score 2, crawling in circles to the right; score 3 , falling down to the right; score 4, failure to walk spontaneously with loss of consciousness.

Measurement of rat cerebral infarct volume. Infarct volume was evaluated $72 \mathrm{~h}$ after obtaining the neurological scores.
Following the sacrifice of the rats through decapitation, the rat brains were rapidly removed, frozen for $20 \mathrm{~min}$ at $-20^{\circ} \mathrm{C}$ and then sectioned at $2-\mathrm{mm}$ intervals from the frontal to the occipital pole in the ischemic hemisphere, generating four coronal slices. 2,3,5-Triphenyltetrazolium chloride (TTC) $(2 \%)$ was subsequently used to stain the slices for $30 \mathrm{~min}$ at $37^{\circ} \mathrm{C}$, and the slices were fixed in $4 \%$ paraformaldehyde overnight. The infarcted lesion area appeared white, in contrast to the red-stained normal areas. A digital scanner was used to capture images of the TTC-stained sections, which were then analyzed using the Motic Images Advanced 3.2 image analysis system (Motic, Hong Kong SAR, China). The degree of brain damage was assessed by measuring brain edema/swelling (13). In order to calculate the infarct zone (IZ) in each brain slice, the area of normal tissue of the ipsilateral hemisphere was subtracted from the area of the contralateral hemisphere; the total IZ, expressed as the percentage of the damaged area relative to the total area of the contralateral hemisphere, was obtained by adding the damaged area of the four slices.

Detection of apoptotic neurons in rat brains. Rat apoptotic neurons in the same infarct area of the ipsilateral cortex were detected $72 \mathrm{~h}$ after obtaining the neurological scores using terminal deoxynucleotidyl transferase-mediated dUTP nick end labeling (TUNEL) fluorescence double staining with a TUNEL kit from Sigma-Aldrich.

Measurement of the GLT-1 and GLAST mRNA levels by reverse transcription-quantitative polymerase chain reaction $(R T-q P C R)$. Rat brains from the control and experimental groups were removed $72 \mathrm{~h}$ after obtaining the neurological scores for the total RNA isolation. RNA was then reverse-transcribed into cDNA. With the synthesized cDNA as a template, primer pairs 5'-AGTATGTGGCGGGCTGCT TCG-3' (upstream) and 5'-GGAAATAATGAGAGGGAG GAT-3' (downstream) for GLT-1; 5'-AACTTTGCCTGTTAC CTTC-3' (upstream) and 5'-CAGTCACAATCTGACCTCC-3' (downstream) for GLAST; and 5'-CGGCAGTCGCCTTGG ACGTT-3' (upstream) and 5'-GCCCTTTCCCATCTCAGC AGCC-3' (downstream) for $\beta$-actin (Primer Express, Applied Biosystems; Life Technologies, Carlsbad, CA, USA)] were used for the qPCR using the ABI Prism ${ }^{\circledR} 7500$ qPCR system (Applied Biosystems). Amplification of $\beta$-actin with $\mathrm{SYBR}^{\circledR}$ Green II-labeled primers was used as an internal control. The data were analyzed using the ABI Prism 7500 SDS software, version 2.0.6 (Applied Biosystems). Quantification was performed using the $2^{-\Delta \Delta \mathrm{ct}}$ method.

Detection of GLT-1 and GLAST protein expression using western blotting. Rat brains were extracted $72 \mathrm{~h}$ after obtaining the neurological scores, and then homogenized and lysed in radioimmunoprecipitation assay buffer containing protease inhibitors (Sigma-Aldrich). The total amount of protein in the brain homogenate was quantified using Biuret colorimetry (Bio-Rad, Hercules, CA, USA). Sodium dodecyl sulfate-polyacrylamide gel (10\%) was used to separate equal amounts $(20 \mu \mathrm{g})$ of protein and electrotransfer them onto the polyvinylidene fluoride membrane (Hybond-C; GE Healthcare, Freiburg, Germany). The membrane was then blocked with 5\% bovine serum albumin for $1 \mathrm{~h}$ and incubated 
Table I. Neurological deficit score and infarct volume of rats 24, 36 and $72 \mathrm{~h}$ after the ischemia-reperfusion injury.

\begin{tabular}{|c|c|c|c|c|c|}
\hline \multirow[b]{2}{*}{ Group } & \multicolumn{4}{|c|}{ Neurological deficit } & \multirow[b]{2}{*}{ Infarct volume, $\mathrm{mm}^{3}(\mathrm{n}=5 /$ group$)$} \\
\hline & $\mathrm{n}$ & $24 \mathrm{~h}$ & $36 \mathrm{~h}$ & $72 \mathrm{~h}$ & \\
\hline Sham & 36 & 0 & 0 & 0 & 0 \\
\hline EPO-sham & 31 & 0 & 0 & 0 & 0 \\
\hline MCAO & 39 & $3.5 \pm 0.4^{\mathrm{a}}$ & $3.1 \pm 0.5^{\mathrm{a}}$ & $2.6 \pm 0.8^{a}$ & $166 \pm 21^{\mathrm{a}}$ \\
\hline ЕРО-MCAO & 34 & $1.5 \pm 0.3^{\mathrm{a}, \mathrm{b}}$ & $1.4 \pm 0.4^{\mathrm{a}, \mathrm{b}}$ & $1.2 \pm 0.6^{\mathrm{a}, \mathrm{b}}$ & $52 \pm 15^{\mathrm{a}, \mathrm{b}}$ \\
\hline
\end{tabular}

${ }^{\mathrm{a}} \mathrm{P}<0.01$ vs. the sham group; ${ }^{\mathrm{b}} \mathrm{P}<0.01$ vs. the $\mathrm{MCAO}$ group. Data are presented as the mean \pm standard deviation. EPO, erythropoietin; $\mathrm{MCAO}$, middle cerebral artery occlusion.

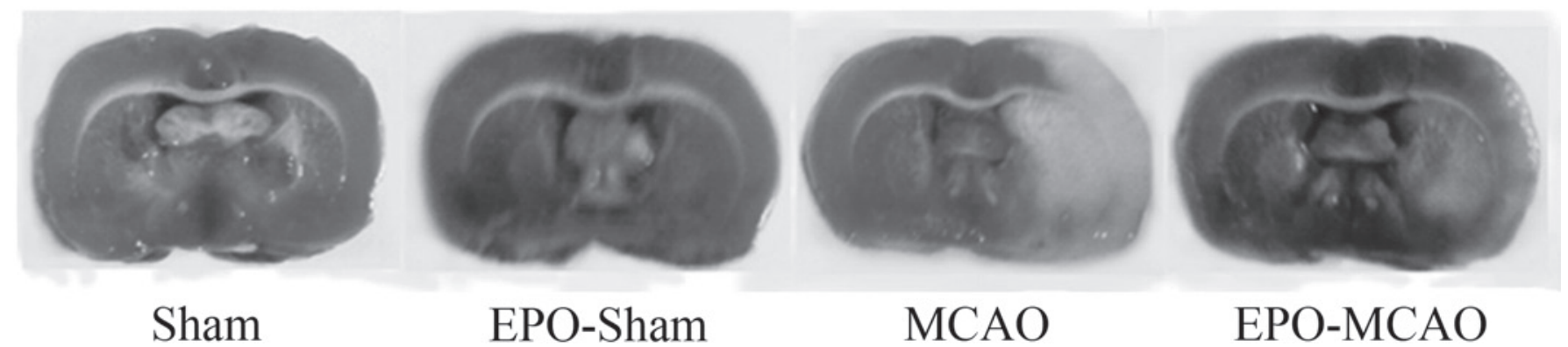

Figure 1. Representative 2,3,5-triphenyltetrazolium chloride staining in coronal sections of different treatment groups. EPO, erythropoietin; MCAO, middle cerebral artery occlusion.

with polyclonal anti-GLT-1 (\#SEE380Mu) and anti-GLAST antibodies (\#SEE806Mu; dilution, 1:1,000; Sigma-Aldrich) for another $2 \mathrm{~h}$ at room temperature. The membrane was washed three times in $1 \mathrm{X}$ phosphate-buffered saline containing $0.5 \%$ Tween 20 (PBST) and then incubated with horseradish peroxidase-labeled anti-mouse secondary antibody (dilution, 1:1,000) for $1 \mathrm{~h}$. The membrane was further washed in PBST three times, for $10 \mathrm{~min}$ each time. Proteins were visualized using an enhanced chemiluminescence protein detection kit (Pierce Biotechnology, Inc., Rockford, IL, USA). The intensity of the bands was analyzed using Scion Image software, version 4.0.3 (Scion Corp., Frederick, MD, USA). $\beta$-actin was used as an internal control for quantifying the protein expression of GLT-1 and GLAST.

Statistical analysis. The number of apoptotic neurons in the corresponding ischemic and non-ischemic sites was counted using a microscope (magnification, $\mathrm{x} 400$ ) by an investigator blinded to the experiment design. The data are expressed as the mean \pm standard deviation and were analyzed using analysis of variance followed by post hoc analysis using the least significant difference test (SPSS 13.0; SPSS Inc., Chicago, IL, USA). $\mathrm{P}<0.05$ was considered to indicate a statistically significant difference.

\section{Results}

EPO preconditioning reduces the infarct volume and improves the neurological function $24 \mathrm{~h}$ after ischemia-reperfusion. The neurological deficit score and infarct volume in the model groups 24, 36 and $72 \mathrm{~h}$ after the ischemia-reperfusion injury were significantly higher than those in the control group $(\mathrm{P}<0.01)$. Pretreatment with EPO significantly reduced the neurological deficit score and infarct volume compared with the MCAO group at the same time-point $(\mathrm{P}<0.01)$ (Fig. 1 and Table I).

EPO preconditioning reduces the number of apoptotic cells $72 h$ after ischemia-reperfusion. More TUNEL-positive apoptotic cells were observed in the IZ of rats in the MCAO group than in the IZ of the control group rats $(\mathrm{P}<0.05)$. The number of TUNEL-positive cells in the EPO-MCAO group was significantly lower than that in the MCAO group $(\mathrm{P}<0.05)$ (Fig. 2).

EPO-preconditioning increases the expression levels of GLT-1 and GLAST mRNA and protein $24 \mathrm{~h}$ after the ischemia. The expression levels of GLT-1 and GLAST mRNA and protein in the MCAO group were significantly reduced $72 \mathrm{~h}$ after the local ischemia compared with those in the control group $(\mathrm{P}<0.01)$. By contrast, EPO-preconditioning significantly increased the GLT-1 and GLAST mRNA and protein expression levels above the levels of the MCAO group ( $\mathrm{P}<0.01)$ (Figs. 3 and 4$)$.

\section{Discussion}

Despite the restoration of blood flow following a period of cerebral ischemia, full cerebral function is not restored in certain stroke patients; instead, reperfusion exacerbates the injury initially caused by the ischemia (14). At present, the most promising approach for the prevention and treatment of ischemic cerebral injury is to address the injury caused by 
A

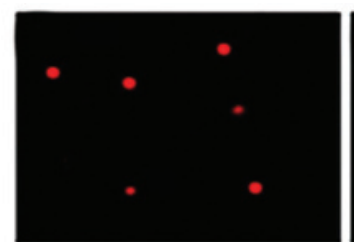

Sham

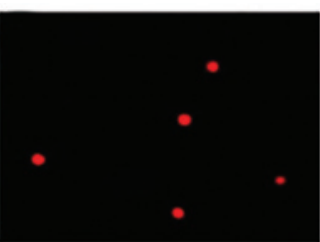

EPO-Sham

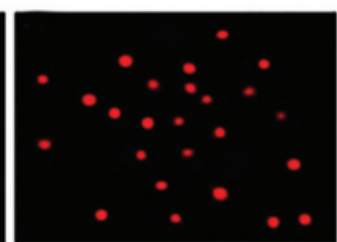

MCAO

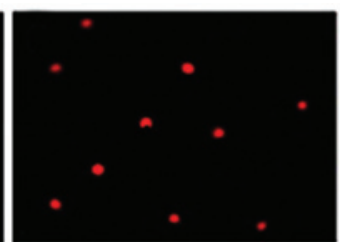

EPO-MCAO

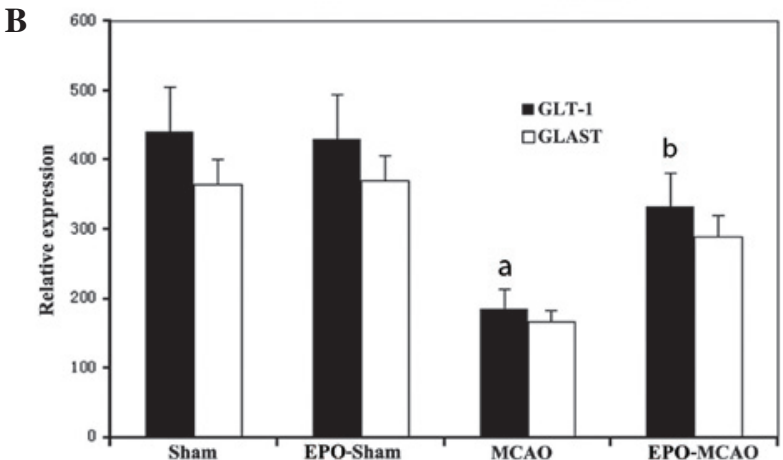

Figure 2. Apoptotic neurons in rat brains were detected by TUNEL fluorescence double staining. (A) Representative TUNEL-positive apoptotic cells in the same infarct area of the ipsilateral cortex of the following groups: Sham $(n=9)$, EPO-sham $(n=8)$, MCAO $(n=10)$ and EPO-MCAO (n=9). (B) Number of apoptotic cells per field in the same infarct area of each group. Data are presented as the mean \pm standard deviation. ${ }^{a} \mathrm{P}<0.01 \mathrm{vs}$. the sham group; ${ }^{\mathrm{b}} \mathrm{P}<0.05 \mathrm{vs}$. the MCAO group at the same reperfusion time. EPO, erythropoietin; MCAO, middle cerebral artery occlusion; GLT-1, glutamate transporter 1; GLAST, glutamate aspartate transporter; TUNEL, terminal deoxynucleotidyl transferase-mediated dUTP nick end labeling.

A

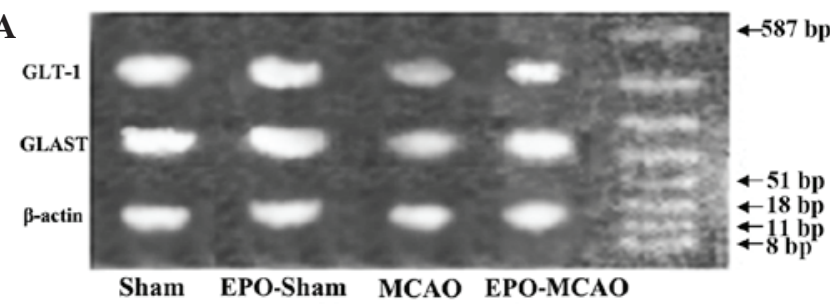

B

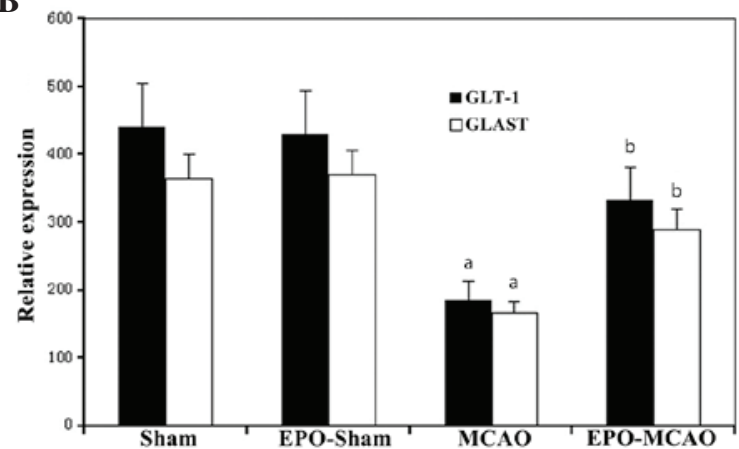

Figure 3. mRNA levels of GLT-1 and GLAST in the sham (n=12), EPO-sham $(\mathrm{n}=10)$, MCAO $(\mathrm{n}=12)$ and EPO-MCAO $(\mathrm{n}=11)$ groups were detected via the reverse transcription polymerase chain reaction following cerebral ischemia/reperfusion. (A) Representative gel photograph of GLT-1 and GLAST mRNA bands in each group. (B) Relative expression values of GLT-1 and GLAST are presented as the mean \pm standard deviation. ${ }^{a} \mathrm{P}<0.01$ vs. the sham group at the same reperfusion time; ${ }^{\mathrm{b}} \mathrm{P}<0.01$ vs. the MCAO group at the same reperfusion time. $\mathrm{EPO}$, erythropoietin; $\mathrm{MCAO}$, middle cerebral artery occlusion; GLT-1, glutamate transporter 1; GLAST, glutamate aspartate transporter.

reperfusion. Curative approaches using medical, physical and biological methods have been successful in animal experiments (15-17); however, not one of these methods has been successfully demonstrated in clinical practice. It is, therefore, of the utmost importance to explore novel therapeutic strategies against ischemia-reperfusion injury.
A
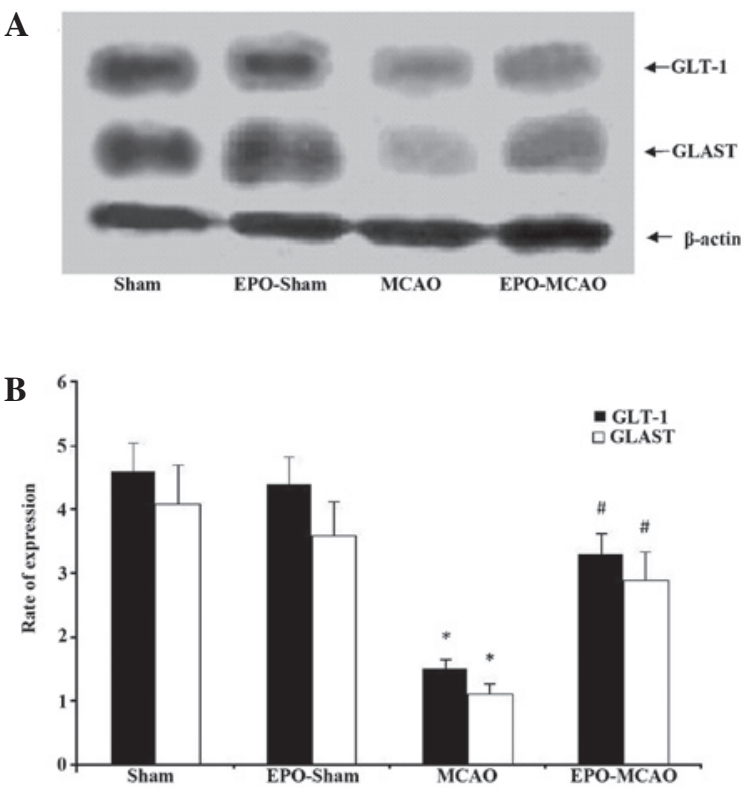

Figure 4. Protein levels of GLT-1 and GLAST proteins in the sham $(n=10)$, EPO-sham $(n=8)$, MCAO $(n=12)$ and EPO-MCAO $(n=9)$ groups were detected via western blotting following cerebral ischemia/reperfusion. (A) Representative protein expression bands of GLT-1 and GLAST in each group. (B) Relative protein expression of GLT-1 and GLAST in the four groups. Data are presented as the mean \pm standard deviation. ${ }^{*} \mathrm{P}<0.01$ vs. the sham group at the same reperfusion time; ${ }^{*} \mathrm{P}<0.01$ vs. the $\mathrm{MCAO}$ group at the same reperfusion time. EPO, erythropoietin; MCAO, middle cerebral artery occlusion; GLT-1, glutamate transporter 1; GLAST, glutamate aspartate transporter.

EPO has been shown to exert a protective effect on cerebral ischemia-reperfusion injury. The protection mediated by EPO may not be due to an increased erythropoietic activity; rather, EPO is believed to inhibit the neuronal apoptosis induced by drugs and traumatic brain injury (11). A study based on liposome-mediated directional transport of EPO to 
the central nervous system revealed that EPO could effectively protect the central nervous system from ischemia-reperfusion injury (18-20). Furthermore, it was found in the present study that EPO pretreatment could significantly reduce the neurological deficit score, infarct volume and number of apoptotic cells following cerebral ischemia-reperfusion. The present results, in combination with previous reports, demonstrated that EPO could be a promising therapeutic drug against cerebral ischemia-reperfusion injury.

Cerebral ischemia-reperfusion injury may involve multiple pathological processes. The release of glutamate, an excitatory neurotransmitter, during cerebral ischemia and hypoxia leads to cerebral injury. Extensive evidence from animal and clinical experiments suggest that high concentrations of extracellular glutamate are closely associated with neural injury (21). Excessive neurotoxicity induced by extracellular glutamate can lead to neuronal death. Under physiological conditions, glutamate is stored in presynaptic vesicles and is released into the synaptic cleft when action potentials arrive, allowing signals to be transmitted to postsynaptic neurons. Generally, glutamate released into the synaptic cleft is transported out of the extracellular space by glutamate transporters (i.e. GLT-1 and GLAST) expressed on astrocytes to reduce the noise-signal ratio and excitatory neurotoxicity. Subsequent to entering the astrocytes, glutamate is converted into glutamine and then transported back into neurons. Under pathological conditions, the transporting activity or expression of the GLT-1 and GLAST decreases, leading to an increased extracellular glutamate concentration that can cause excitatory neurotoxicity (21). GLT-1 and GLAST are also expressed in other types of cells besides astrocytes, including neurons, but only a small quantity of glutamate is imported into these cells via these transporters (22). Furthermore, glutamate can be transported in a bidirectional manner under pathological conditions, which can result in a net transport of glutamate from the inside to the outside of the cell (23). Overexpression of GLT-1 can markedly reduce hypoxia-induced cerebral injury (9).Specific overexpression of GLT-1 in astrocytes under the control of a glial fibrillary acidic protein promoter showed that GLT-1 protected the neurons in the brain slices that were under oxygen and glucose deprivation conditions (24). EPO has been found to exert its neuroprotection by stimulating Janus kinase 2 phosphorylation, which leads to the activation of signal transducer and activator of transcription 5, Akt and nuclear factor- $\kappa \mathrm{B}$ pathways and initiates the expression of neuroprotective proteins, such as glutamate transporters (e.g. GLT-1 and GLAST) (25-28). Of note, the present results showed that cerebral ischemia-reperfusion reduced the mRNA and the protein levels of GLT-1 and GLAST, whereas EPO preconditioning significantly increased these levels following ischemia-reperfusion. This suggests that the protection against cerebral ischemia-reperfusion injury, as a result of EPO preconditioning, may be partly due to the upregulation of GLT-1 and/or GLAST expression; however, since GLT-1 and GLAST are expressed in both astrocytes and other cells, the cell type that is involved in the protective role of EPO against cerebral ischemia-reperfusion injury remains to be elucidated. In addition, the occlusion in the right side of the brain in the MCAO model could affect the blood brain barrier and allow EPO to also enter the left side of the brain and upregulate the
GLT-1 and GLAST expression in general. Thus, the exact effects and mechanisms of EPO on MCAO-induced alterations in GLT-1 and GLAST require further study using the contralateral side of the brain as a control.

In conclusion, EPO pretreatment effectively relieved acute cerebral ischemia-reperfusion injury by reducing the neurological deficit score, infarct volume and number of apoptotic cells. This finding may be associated with the increased expression and transport activity of GLT-1 and GLAST. Further studies are necessary in order to fully comprehend the detailed molecular mechanisms underlying the protective effects of EPO preconditioning against cerebral ischemia-reperfusion injury.

\section{References}

1. Koudstaal PJ, Stibbe J and Vermeulen M: Fatal ischaemic brain oedema after early thrombolysis with tissue plasminogen activator in acute stroke. BMJ 297: 1571-1574, 1988.

2. Clark RK, Lee EV, White RF, Jonak ZL, Feuerstein GZ and Barone FC: Reperfusion following focal stroke hastens inflammation and resolution of ischemic injured tissue. Brain Res Bull 35: 387-392, 1994.

3. Mitani A and Kataoka K: Critical levels of extracellular glutamate mediating gerbil hippocampal delayed neuronal death during hypothermia: Brain microdialysis study. Neuroscience 42: 661-670, 1991.

4. Keelan J, Bates TE and Clark JB: Differences in the amount of glutamate released by neonatal and adult synaptosomes under conditions of in vitro ischaemia/reperfusion. Biochem Soc Trans 24: 425S, 1996.

5. Semkova I, Schilling M, Henrich-Noack P, Rami A and Krieglstein J: Clenbuterol protects mouse cerebral cortex and rat hippocampus from ischemic damage and attenuates glutamate neurotoxicity in cultured hippocampal neurons by induction of NGF. Brain Res 717: 44-54, 1996.

6. Perego C, Vanoni C, Bossi M, Massari S, Basudev H, Longhi R and Pietrini G: The GLT-1 and GLAST glutamate transporters are expressed on morphologically distinct astrocytes and regulated by neuronal activity in primary hippocampal cocultures. J Neurochem 75: 1076-1084, 2000.

7. Fukamachi S, Furuta A, Ikeda T, Ikenoue T, Kaneoka T, Rothstein JD and Iwaki T: Altered expressions of glutamate transporter subtypes in rat model of neonatal cerebral hypoxiaischemia. Brain Res Dev Brain Res 132: 131-139, 2001.

8. Han BC, Koh SB, Lee EY and Seong YH: Regional difference of glutamate-induced swelling in cultured rat brain astrocytes. Life Sci 76: 573-583, 2004

9. Harvey BK, Airavaara M, Hinzman J, Wires EM, Chiocco MJ, Howard DB, Shen H, Gerhardt G, Hoffer BJ and Wang Y: Targeted over-expression of glutamate transporter 1 (GLT-1) reduces ischemic brain injury in a rat model of stroke. PLoS One 6: e22135, 2011

10. Calapai G, Marciano MC, Corica F, Allegra A, Parisi A, Frisina N, Caputi AP and Buemi M: Erythropoietin protects against brain ischemic injury by inhibition of nitric oxide formation. Eur J Pharmacol 401: 349-356, 2000.

11. Tang Z, Sun X, Shi Q, Wang X, Xie Y, Huo G, Zhou S and Liao Z: Beneficial effects of carbamylated erythropoietin against oxygen-glucose deprivation/reperfusion-induced astrocyte swelling: Proposed molecular mechanisms of action. Neurosci Lett 530: 23-28, 2012.

12. Longa EZ, Weinstein PR, Carlson S and Cummins R: Reversible middle cerebral artery occlusion without craniectomy in rats. Stroke 20: 84-91, 1989.

13. Shimakura A, Kamanaka Y, Ikeda Y, Kondo K, Suzuki Y and Umemura K: Neutrophil elastase inhibition reduces cerebral ischemic damage in the middle cerebral artery occlusion. Brain Res 858: 55-60, 2000.

14. Patt A, Rutherford RB, Pearce WH and Repine JE: Cerebral ischemia-reperfusion injury in the gerbil. J Surg Res 42: 462-466, 1987.

15. Tausompos C and Panoulis C: Tauoutouzas K, Zetaografos G and Papalois A: The effect of the antioxidant drug "U-74389G" on oophoritis during ischemia reperfusion injury in rats. Antiinflamm Antiallergy Agents Med Chem 13: 103-107, 2014. 
16. Lehrke $M$ and Lebherz C: AAV-mediated gene therapy for atherosclerosis. Curr Atheroscler Rep 16: 434, 2014.

17. Schlegel A, Kron P, Graf R, Dutkowski P and Clavien PA: Warm vs. cold perfusion techniques to rescue rodent liver grafts. J Hepatol 61: 1267-1275, 2014.

18. Brines ML, Ghezzi P, Keenan S, Agnello D, de Lanerolle NC, Cerami C, Itri LM and Cerami A: Erythropoietin crosses the blood-brain barrier to protect against experimental brain injury. Proc Natl Acad Sci USA 97: 10526-10531, 2000.

19. Shin T, Ahn M, Moon C and Kim S: Erythropoietin and autoimmune neuroinflammation: Lessons from experimental autoimmune encephalomyelitis and experimental autoimmune neuritis. Anat Cell Biol 45: 215-220, 2012.

20. McCook O, Georgieff M, Scheuerle A, Möller P, Thiemermann C and Radermacher P: Erythropoietin in the critically ill: Do we ask the right questions? Crit Care 16: 319, 2012.

21. Grewer C, Gameiro A, Zhang Z, Tao Z, Braams S and Rauen T: Glutamate forward and reverse transport: From molecular mechanism to transporter-mediated release after ischemia. IUBMB Life 60: 609-619, 2008

22. Akbar MT, Torp R, Danbolt NC, Levy LM, Meldrum BS and Ottersen OP: Expression of glial glutamate transporters GLT-1 and GLAST is unchanged in the hippocampus in fully kindled rats. Neuroscience 78: 351-359, 1997.
23. Persson M and Rönnbäck L: Microglial self-defence mediated through GLT-1 and glutathione. Amino Acids 42: 207-219, 2012.

24. Romera C, Hurtado O, Mallolas J, Pereira MP, Morales JR, Romera A, Serena J, Vivancos J, Nombela F, Lorenzo P, et al: Ischemic preconditioning reveals that GLT1/EAAT2 glutamate transporter is a novel PPARgamma target gene involved in neuroprotection. J Cereb Blood Flow Metab 27: 1327-1338, 2007.

25. Digicaylioglu M and Lipton SA: Erythropoietin-mediated neuroprotection involves cross-talk between Jak2 and NF-kappaB signalling cascades. Nature 412: 641-647, 2001.

26. Um M and Lodish HF: Antiapoptotic effects of erythropoietin in differentiated neuroblastoma SH-SY5Y cells require activation of both the STAT5 and AKT signaling pathways. J Biol Chem 281: 5648-5656, 2006.

27. Byts N, Samoylenko A, Fasshauer T, Ivanisevic M, Hennighausen L, Ehrenreich H and Sirén AL: Essential role for Stat5 in the neurotrophic but not in the neuroprotective effect of erythropoietin. Cell Death Differ 15: 783-792, 2008.

28. Hosseinzadeh Z, Bhavsar SK, Sopjani M, Alesutan I, Saxena A, Dërmaku-Sopjani M and Lang F: Regulation of the glutamate transporters by JAK2. Cell Physiol Biochem 28: 693-702, 2011. 